\title{
Experimental Study on the Mechanism of Hypoglycemic Action of sulfonylurea
}

By

\section{Assist.-Professor Hideo YOSHIDA M.D., Sadami YOKOO M.D. Osamu AOCHI and Tomio UEHIRA}

Medical Department of Kyoto Prefectural Medical School (Director: Professor S. Tateishi M.D.)

By the discovery of insulin ${ }^{1)}$ in 1922, the therapy of diabetes mellitus was improved very much and the mortality of diabetes mellitus is very few. But we have no view of complete recovering. From this long course per oral medicine was expected very much.

In 1942 Janbon $^{2}$ discovered the hypoglycemic action of IPTD and now there is no one who suspects the hypoglycemic effect of sulfonylurea. However, about the mechanism of hypoglycemic action of this medicine, there are many different opinions in the researchers throughout the world.

In this congress of Japanese Society of Internal Medicine one of us Yoshida take part in simposium of therapy of diabetes mellitus. So we study on the mechanism of thypoglycemic action of sulfonylurea. We have the following results.

\section{Method}

Many rabbits and rats were used. We used Wahrburg's pressure measure method and incubate in $38^{\circ} \mathrm{C} 1$ hour long. We used buffer solution $\mathrm{pH} 7.4$ with $0.9 \% \mathrm{NaCl}$ solution and $0.1 \mathrm{Mol}$ potassium-phosphate dibasic and we added glucose powder in this buffer solution to $150 \sim 200 \mathrm{mg} / \mathrm{dl}$. Glucose was measured with Hagedorn-Jensen method.

On the histological study we used pironin-methylgreen, Gomori and Schiff-PAS method besides haematoxilin-eosin and urtra violet microscope (2650 ̊) and electronmicroscope.

\section{Results}

A) The effect of sulfonylurea on the blood sugar

1) The blood sugar of normal rabbits, which were given BZ55 pro Kg. 0.25g, first a little ascends, next descends ond after a long time comes back to original value. (Fig. 1) There are some individual variations of this ascendence and descendence on the time or the value. Many of them ascend after about 15 minutes, and after 30 minutes they show the highest value, namely the original value $80 \mathrm{mg} / \mathrm{dl}$ becomes $100 \mathrm{mg} / \mathrm{dl}$. After an hour many of them come back to the original value. After about 3 hours from the beginning the blood sugar descends and from 3 hours to 5 hours we see the lowest value about $50 \mathrm{mg} / \mathrm{dl}$. Then the blood sugar ascends gradually and after 24 hours it shows generally no influence. There is clear difference between natural descendence of normal rabbits and the curve of the rabbits which were given $\mathrm{BZ} 55$. 


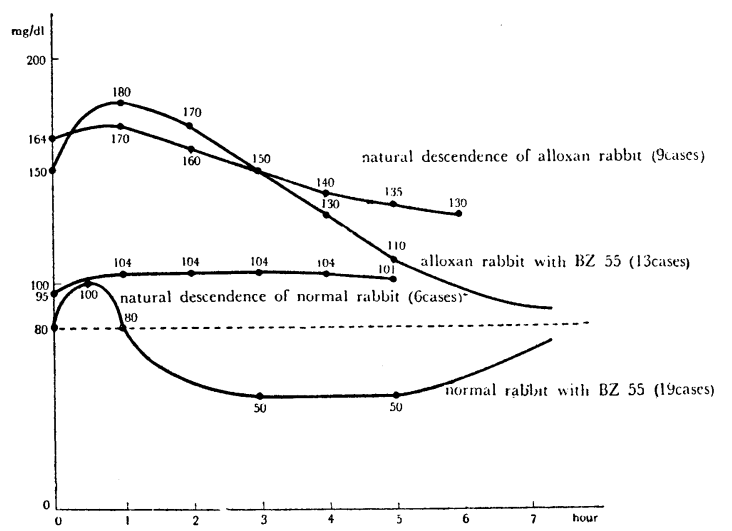

Fig. 1. The effect of sulfonylurea on the blood sugar
2) When sulfonylurea is given to alloxan rabbits, the blood sugar, first a little ascends, next descends and after a long time comes back to original value as the normal rabbits. But the descendence of the blood sugar might be later than normal. When first the blood sugar shows $150 \mathrm{mg} / \mathrm{dl}$ it ascends to $180 \mathrm{mg} / \mathrm{dl}$ after about an hour. After 3 hours it descends to $150 \mathrm{mg} / \mathrm{dl}$. Then the blood sugar of alloxan rabbits might descend to nearly normal value. This time too, clear difference is re-

cognised from natural descendence of alloxan rabbits.

When the blood sugar of alloxan rabbits already descends to normal value, they show about the same wave as normal rabbits.

3) While, when every 10 days BZ 55 or D 860 per os $0.05 \sim 0.2 \mathrm{~g}$ is piven, or every 30 days BZ 55 subcutan $0.1 \mathrm{~g}$ is given, blood sugar is about normal value after 24 hours of latest medicine.

4) The effect of the sulfonylurea on the blood sugar, when $3 g$ at a time are given to normal person.

At 9.00 a. m. blood sugar is measured in hunger condition and immediately BZ $553 \mathrm{~g}$ are given. After 15 minutes, 30 minutes, 1 hour and 3 hours blood sugar is measured. Next day we measure the same course without medicine as control. On normal persons in hunger condition there is no difference between 9. a. m. and 12 a. m. After 3 hours of BZ 55 blood sugar increases to about $80 \%(65.9 \sim 91.4 \%)$ of it at 9 a. m.

But at the time of BZ 55 giving, it becomes about 65\% (49.5 76.0\%). But at this time we can see rarely a person, who shows no difference with or without medicine. (see Tab. 1,2)

B) The effect of sulfonylurea on the carbohydrate metabolism in vitro.

1) Carbohydrate metabolism of rabbit which is given sulfonylurea. Carbohydrate metabolism of those rabbits which are given BZ 55 pro Kg. $0.25 \mathrm{~g}$ is measured. (see Tab. 3)

On the studium of ascendence of blood sugar, output of liver glucose increases and on the studium of maximum blood sugar or descendence of blood sugar output of liver glucose decreases. On the studium of descendence of blood sugar uptake of diaphragm glucose inclines to increase.

2) The effect of serum of rabbits, which are given sulfonylurea, on output of liver glucose or glucose uptake of diaphragm of normal rats. We measured carbohydrate metabolism of normal rats in the medium of glucose-buffer $1.5 \mathrm{cc}$ and serum $0.5 \mathrm{cc}$ of rats, which are given BZ 55 pro Kg. 0.25 g. Serum of ascendence studium of blood sugar in- 


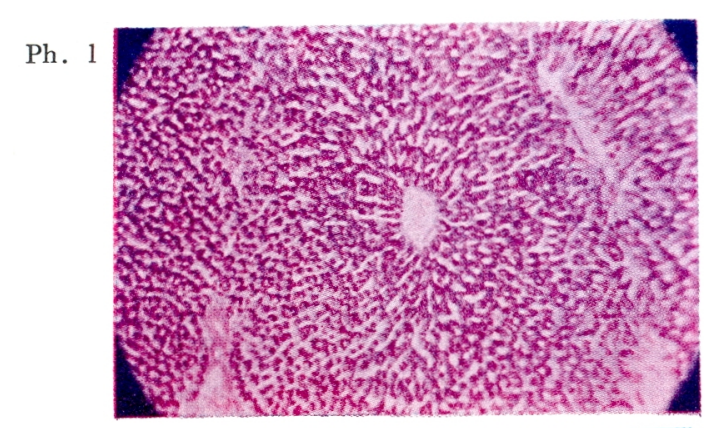

Ph. 2

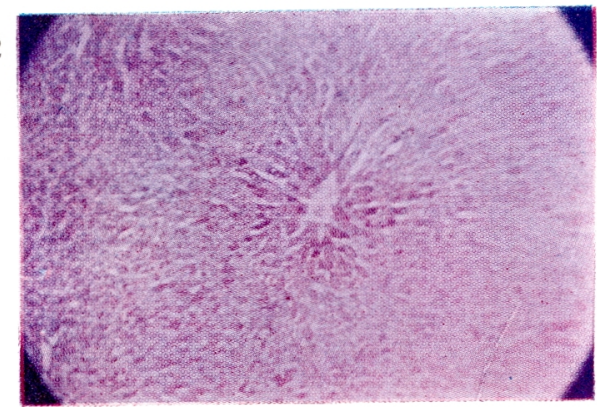

$\mathrm{Ph} .3$

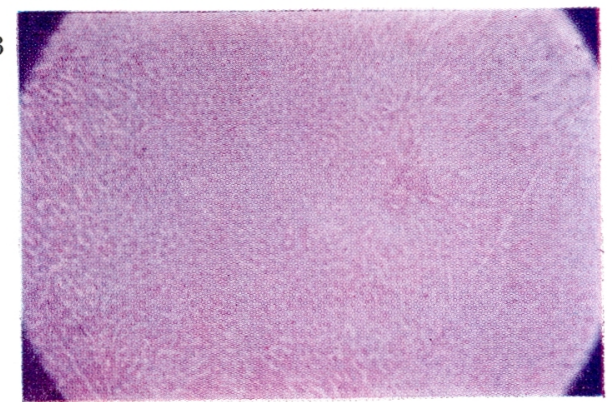

Ph. 4

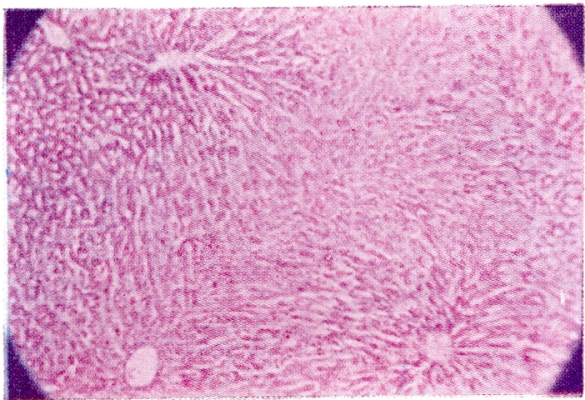

Ph. 5

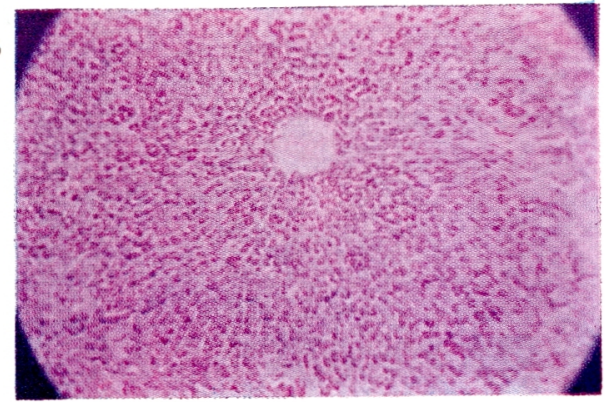

Ph. 6

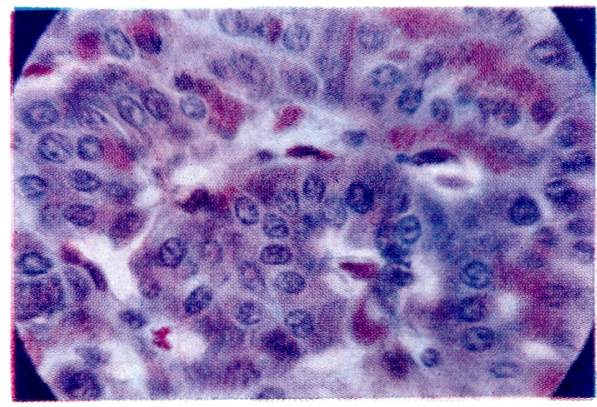

$\mathrm{Ph}$.

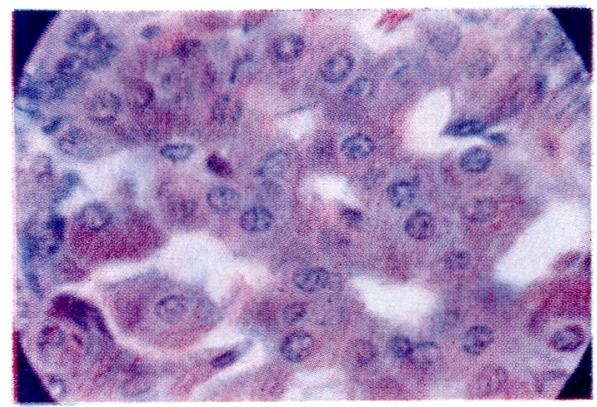

$\mathrm{Ph}$.

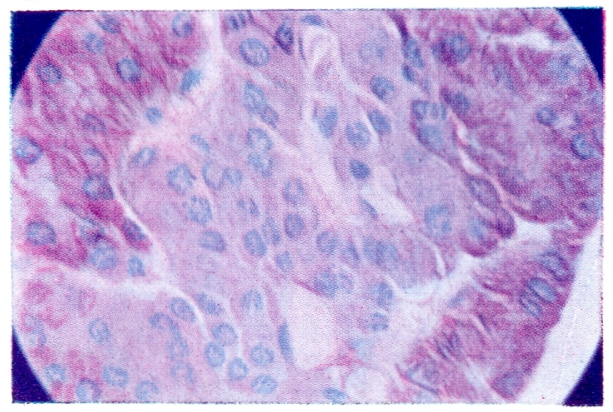

Ph. 9

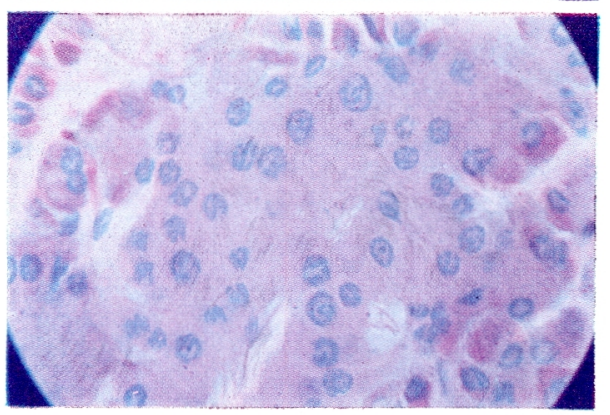

Ph. 10

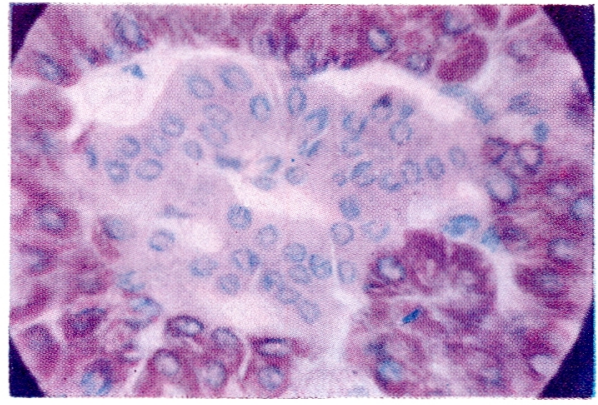



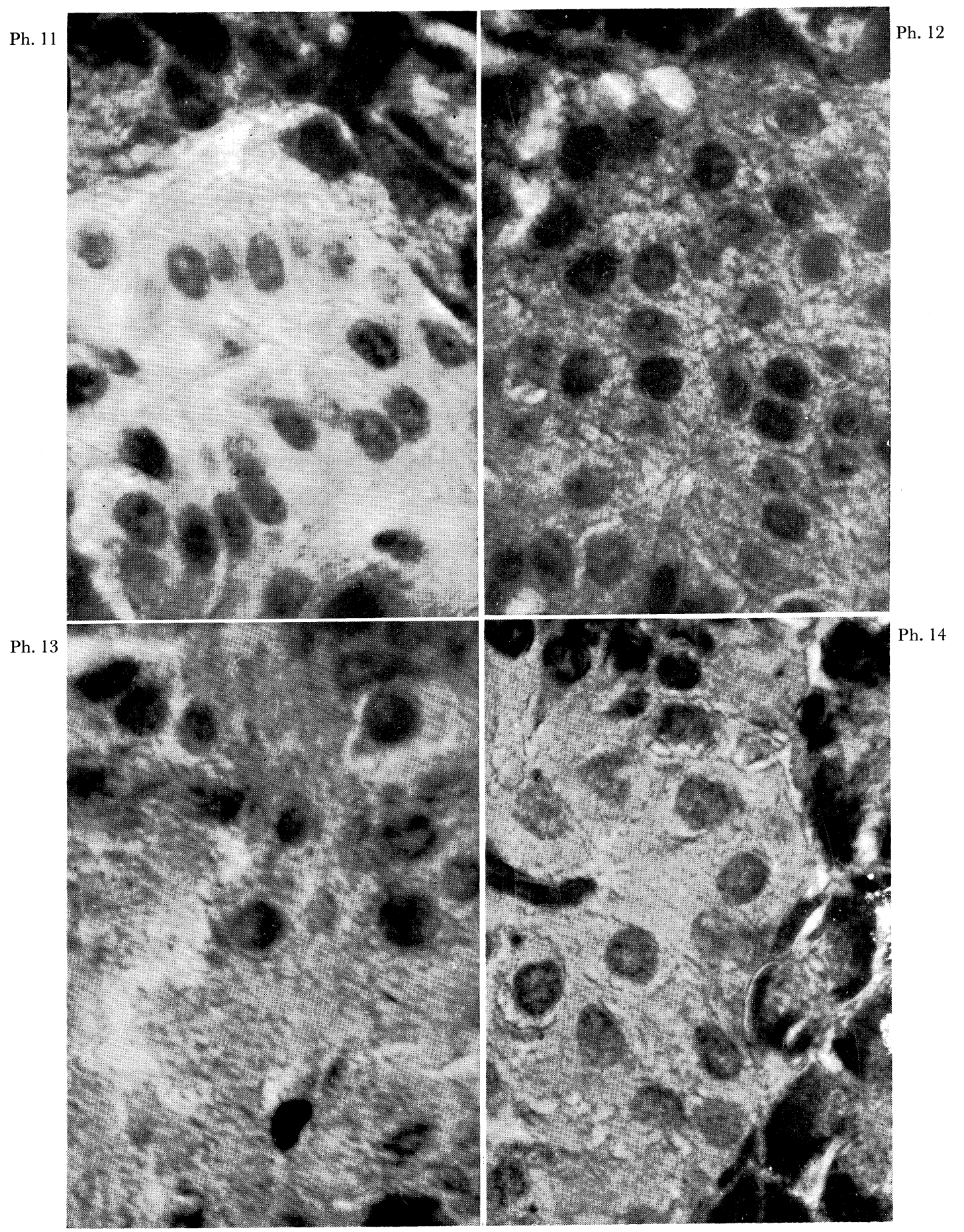
Ph. 15

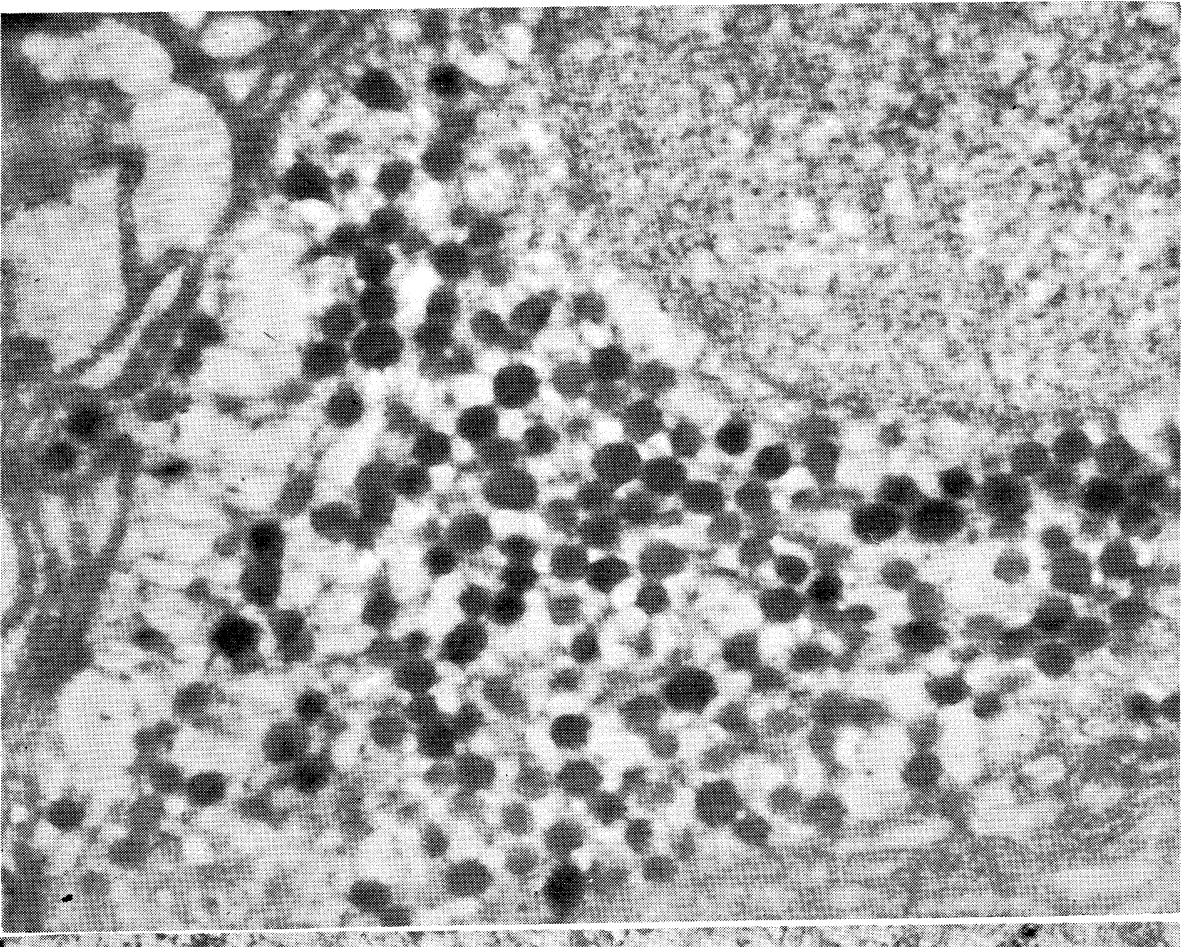

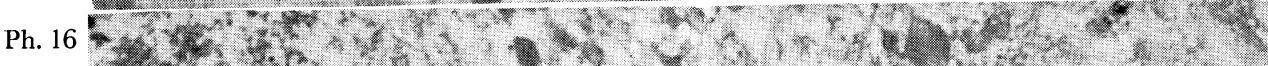

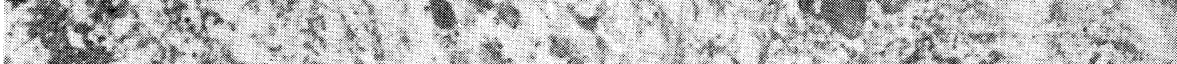

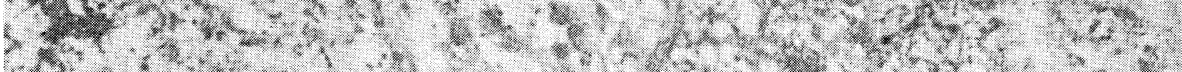

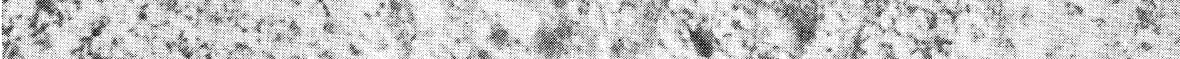

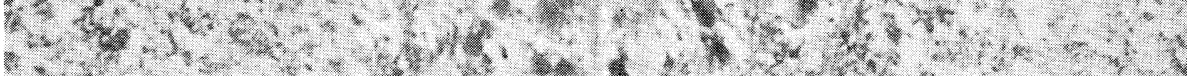

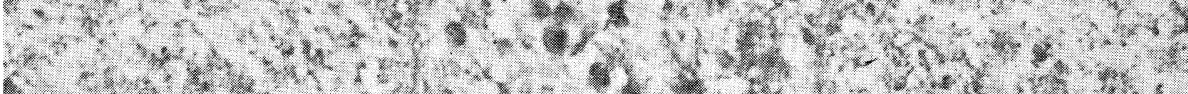

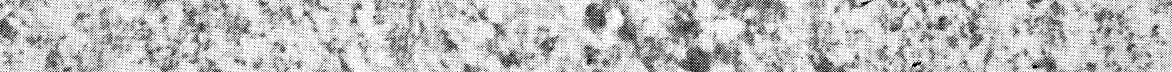

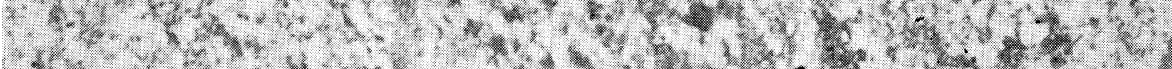

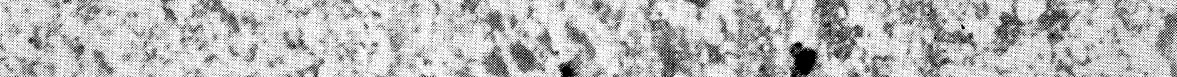

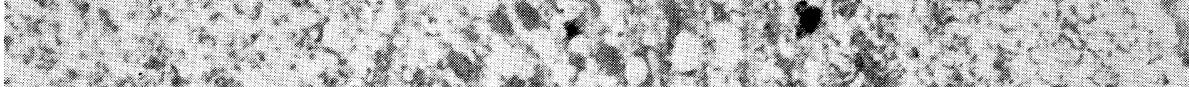

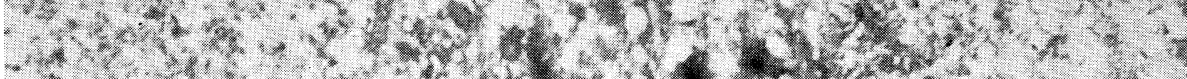

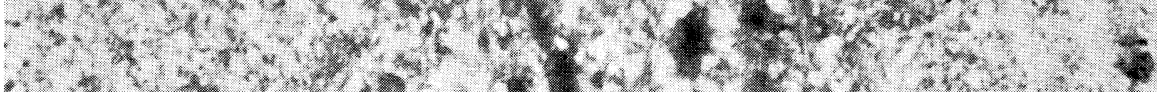

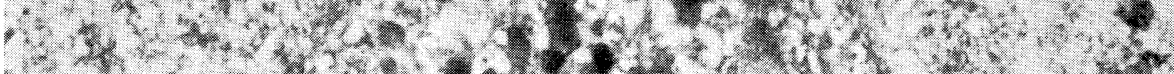

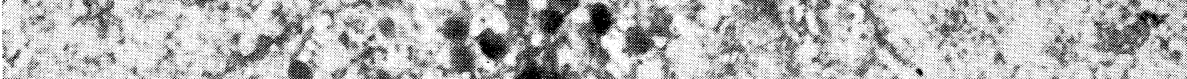

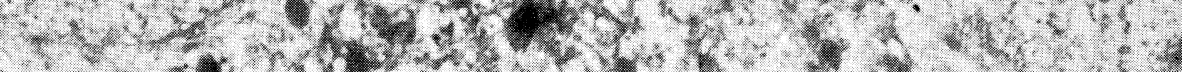

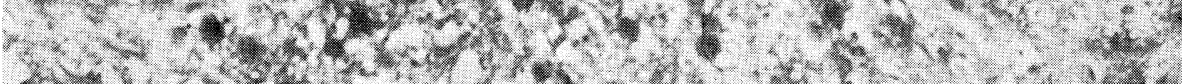

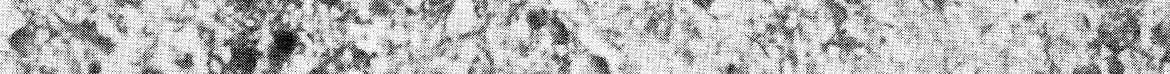

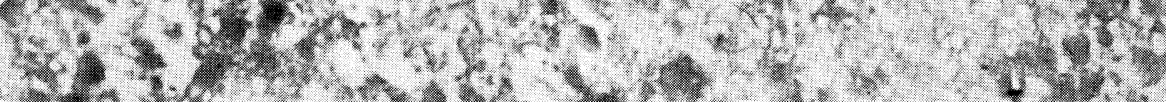

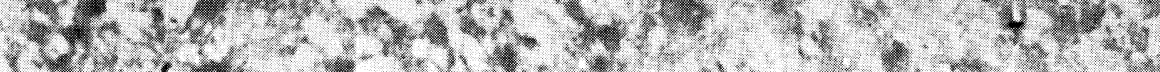

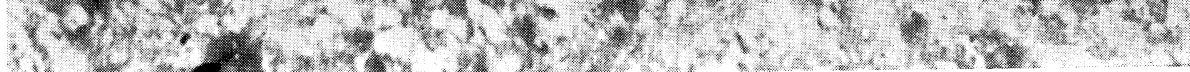




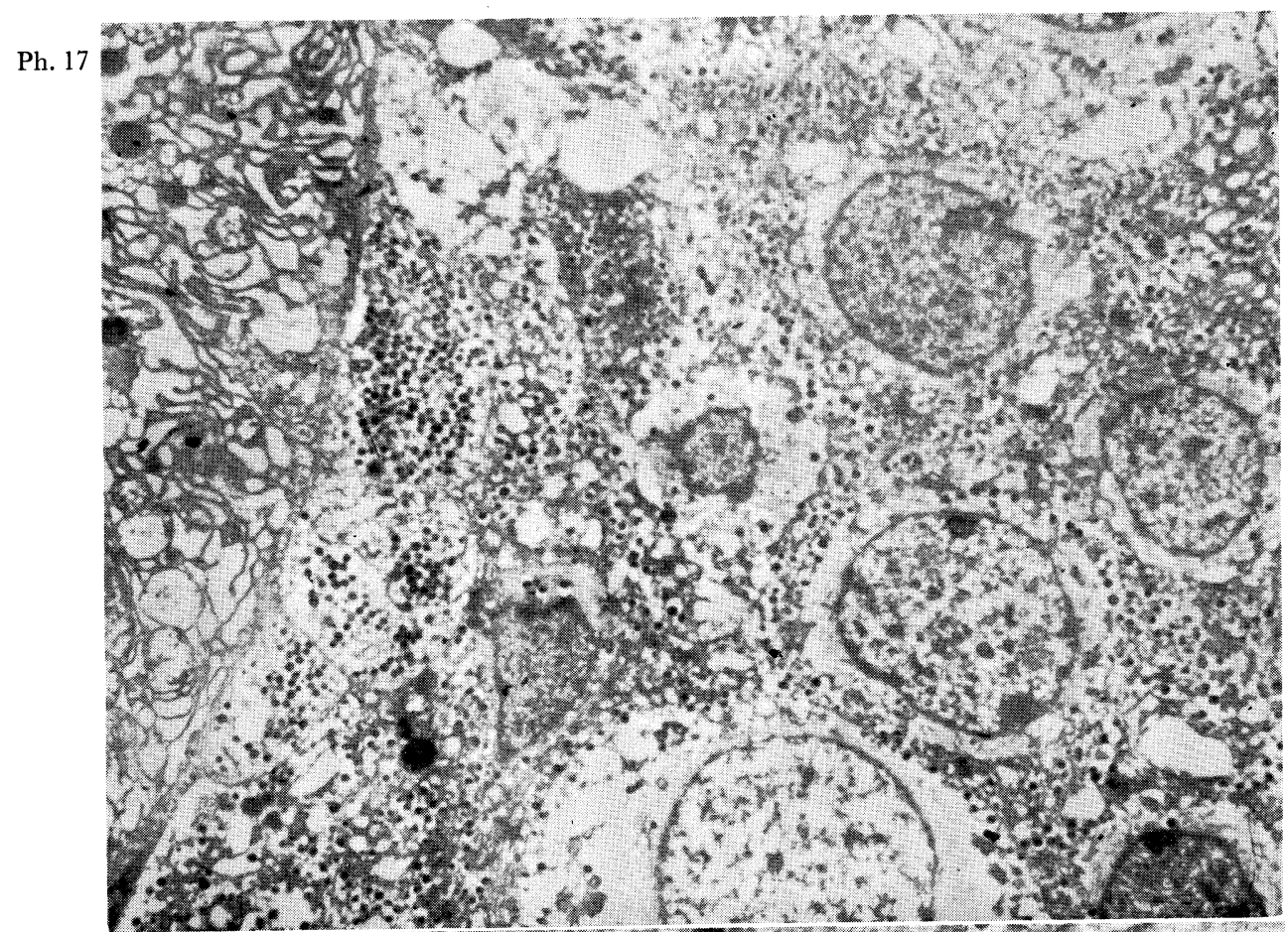

Ph. 18

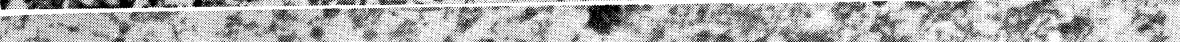

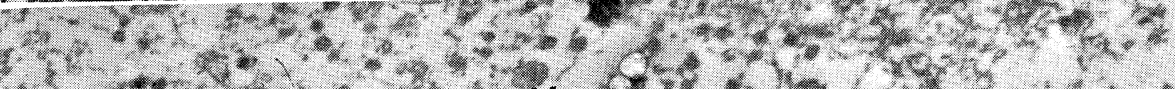

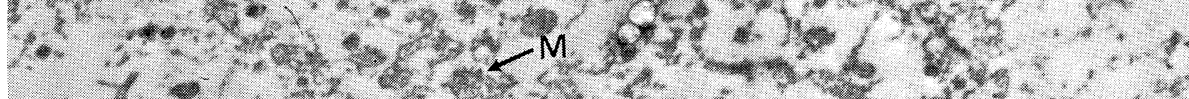

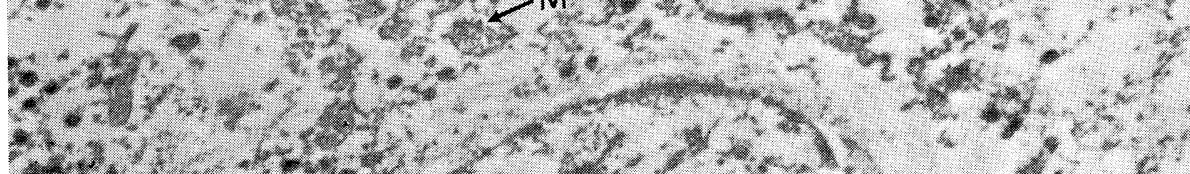

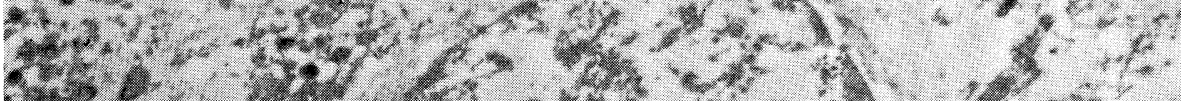

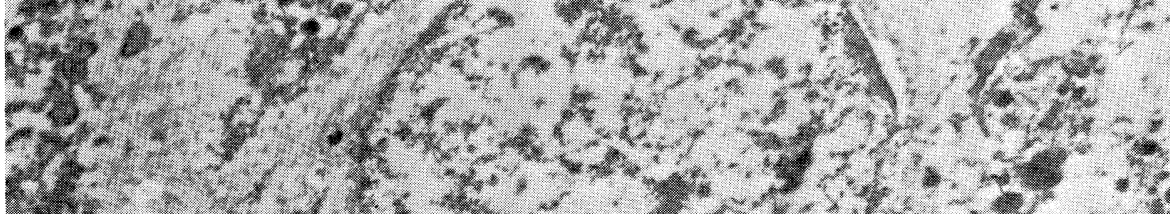

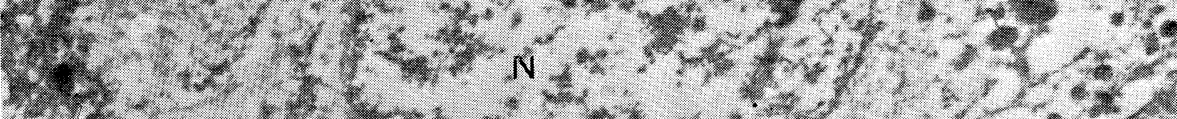

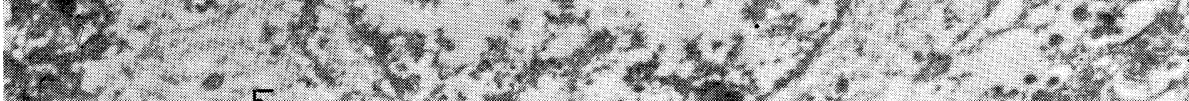

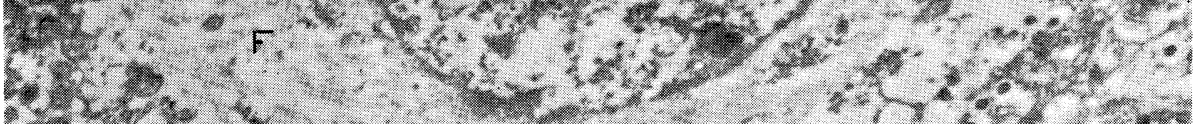

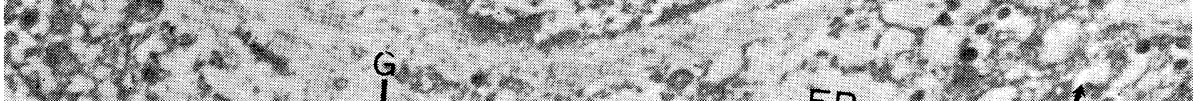
2 2. 2.306.

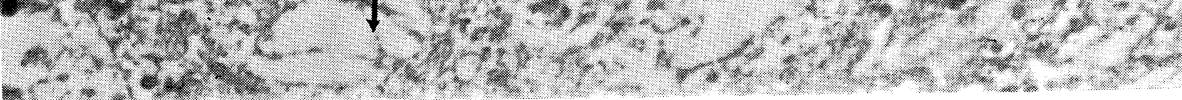


Table 1. Blood sugar of normal persons giving BZ 55 3g.

\begin{tabular}{|c|c|c|c|c|c|c|c|c|}
\hline Normal & Medicine & first & $15 \mathrm{~min}$. & $30 \mathrm{~min}$. & 1 hour & 3 hours & $\frac{3 \text { hours }}{\text { first }}$ & $\frac{\text { minimum }}{\text { first }}$ \\
\hline 1 & $\mathrm{BZ} 553 \mathrm{~g}$ & 91 & 75 & 57 & 67 & 63 & $69.2 \%$ & $62.1 \%$ \\
\hline 2 & "I & 75 & 76 & 62 & 57 & 61 & $81.3 \%$ & $76.0 \%$ \\
\hline 3 & "l & 76 & 73 & 62 & 66 & 65 & $85.5 \%$ & $81.6 \%$ \\
\hline 4 & " & 69 & 69 & 64 & 59 & 64 & $92.7 \%$ & $85.5 \%$ \\
\hline 5 & $" 1$ & 112 & 87 & 74 & 77 & 69 & $61.6 \%$ & $61.6 \%$ \\
\hline 6 & " & 95 & 83 & 92 & 95 & 92 & $96.8 \%$ & $96.8 \%$ \\
\hline 7 & " & 81 & 80 & 81 & 73 & 74 & $91.3 \%$ & $90.1 \%$ \\
\hline 8 & " & 79 & 70 & 46 & 54 & 75 & $94.9 \%$ & $58.2 \%$ \\
\hline 9 & " & 83 & 83 & 76 & 78 & 72 & $86.7 \%$ & $86.7 \%$ \\
\hline Medium & BZ $553 \mathrm{~g}$ & 85 & 78 & 69 & 70 & 71 & $84.4 \%$ & $77.6 \%$ \\
\hline 1 & 0 & 74 & 71 & 71 & 78 & 73 & $98.6 \%$ & $95.9 \%$ \\
\hline 2 & 0 & 57 & 63 & 63 & 63 & 64 & $112.2 \%$ & $110.5 \%$ \\
\hline 3 & 0 & 68 & 72 & 72 & 68 & 68 & $100.0 \%$ & $100.0 \%$ \\
\hline 4 & 0 & 70 & 65 & 73 & 70 & 73 & $104.2 \%$ & $92.8 \%$ \\
\hline 5 & 0 & 90 & 87 & 76 & 87 & 90 & $100.0 \%$ & $84.4 \%$ \\
\hline 6 & 0 & 87 & 83 & 90 & 90 & 95 & $109.1 \%$ & $95.4 \%$ \\
\hline 7 & 0 & 44 & 47 & 49 & 53 & 49 & $111.3 \%$ & $106.8 \%$ \\
\hline 8 & 0 & 61 & 57 & 56 & 61 & 66 & $108.2 \%$ & $91.8 \%$ \\
\hline 9 & 0 & 76 & 78 & 74 & 81 & 74 & $97.4 \%$ & $97.4 \%$ \\
\hline Medium & 0 & 70 & 69 & 69 & 72 & 72 & $104.5 \%$ & $97.2 \%$ \\
\hline
\end{tabular}

creases the glucose output of liver of other animals. Serum of descendence studium increases the glucose uptake of diaphragm. Each method can be thought a quantitative analysis of glucagon ${ }^{\left.3)^{4) 5} 6\right)}$ or insulin. (see Tab. 4)

3) The effect of sulfonylurea in vitro on the glucose uptake of diaphragm.

When glucose uptake of diaphragm is measured, if insulin in various concentration is added, glucose uptake of diaphragm rises including insulin concentration. ${ }^{7)}$ (Fig. 2) When glucose uptake of diaphragm is $17 \mathrm{mg} /$ dry weight $1 \mathrm{~g}$ without insulin, this uptake will be $40 \mathrm{mg} / \mathrm{dry}$ weight $1 \mathrm{~g}$ with $1 \mathrm{u} . / \mathrm{cc}$ insulin.

But the glucose uptake of diaphragm does not rise with sulfonylurea in vitro. (see Tab. 5)

4) The influence of sulfonylurea in vitro on the effect of insulin on the carbohydrate metabolism of diaphragm.

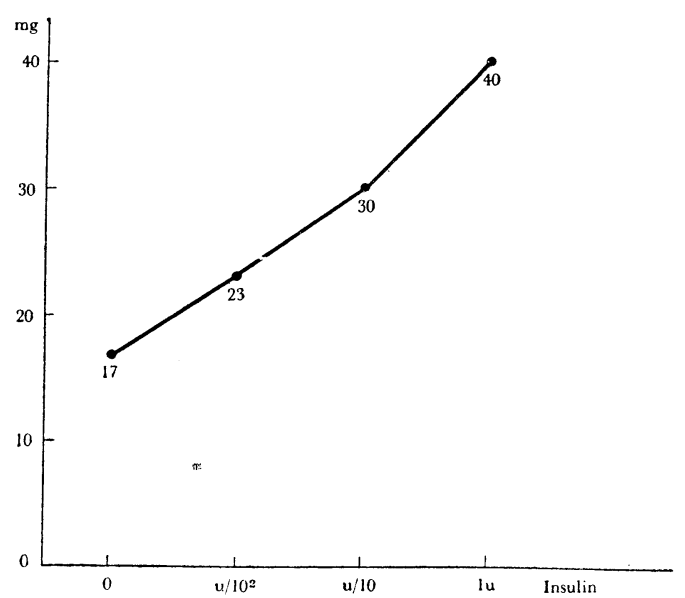

Fig. 2. The effect of insulin on the uptake of dirphragm 
Table 2. Blood sugar of diabetics giving BZ $553 \mathrm{~g}$.

\begin{tabular}{|c|c|c|c|c|c|c|c|}
\hline Diabetics & Medicine & first & $15 \mathrm{~min}$. & $30 \mathrm{~min}$. & 1 hour & 3 hours & $\frac{3 \text { hours }}{\text { first }}$ \\
\hline 1 & BZ $553 \mathrm{~g}$ & 188 & 178 & 172 & 172 & 100 & $53.2 \%$ \\
\hline 2 & "1 & 265 & 269 & 261 & 253 & 216 & $81.5 \%$ \\
\hline 3 & "1 & 116 & 111 & 111 & 94 & 78 & $67.2 \%$ \\
\hline 4 & "1 & 354 & 345 & 353 & 331 & 270 & $76.2 \%$ \\
\hline 5 & "I & 322 & 312 & 302 & 284 & 234 & $72.6 \%$ \\
\hline 6 & "I & 150 & 150 & 150 & 146 & 107 & $71.3 \%$ \\
\hline 7 & "1 & 137 & 133 & 84 & 77 & 68 & $49.6 \%$ \\
\hline 8 & "I & 368 & 367 & 353 & 326 & 209 & $56.8 \%$ \\
\hline 9 & 11 & 141 & 134 & 115 & 96 & 60 & $42.6 \%$ \\
\hline Medium & BZ $553 \mathrm{~g}$ & 227 & 222 & 211 & 198 & 149 & $63.4 \%$ \\
\hline 1 & 0 & 146 & 142 & 142 & 135 & 124 & $65.9 \%$ \\
\hline 2 & 0 & 266 & 262 & 257 & 251 & 214 & $80.4 \%$ \\
\hline 3 & 0 & 82 & 75 & 77 & 82 & 75 & $91.4 \%$ \\
\hline 4 & 0 & 379 & 465 & 441 & 434 & 334 & $88.1 \%$ \\
\hline 5 & 0 & 336 & 338 & 336 & 322 & 273 & $81.2 \%$ \\
\hline 6 & 0 & 149 & 142 & 136 & 136 & 117 & $78.5 \%$ \\
\hline 7 & 0 & 129 & 126 & 124 & 98 & 89 & $69.0 \%$ \\
\hline 8 & 0 & 262 & 250 & 239 & 234 & 198 & $75.5 \%$ \\
\hline 9 & 0 & 83 & 79 & 74 & 83 & 81 & $97.6 \%$ \\
\hline Medium & 0 & 204 & 209 & 203 & 197 & 167 & $80.8 \%$ \\
\hline
\end{tabular}

When glucose uptake of diaphragm is measured, insulin $0.1 \mathrm{u} / \mathrm{cc}, \mathrm{BZ} 551 \mathrm{mg} / \mathrm{cc}$ and both were added in vitro. The effect of insulin on the glucose up take of diaphragm does not rise by the addition of sulfonylurea in vitro. (see Tab. 6)

C) The influence of sulfonylurea on the liver glycogen of rabbbits.

With Schiff-PAS method we observed liver glycogen of rabbits, which were given per os BZ 55 pro Kg. $0.25 \mathrm{~g}$, at 15 minutes, 30 minutes, 1.5 hours and 3 hours after giving.

On the studium of ascendence of blood sugar liver glycogen decreases and there is almost no liver glycogen on the studium of hyperglycemy.

On the studium of descendence of blood sugar liver glycogen increases gradually. This decrease of liver glycogen begins on periphery of liver acinus and the increase begins on central part. (see Ph. 1. normal, liverglycogen much; Ph. 2. after 15m., decrease; Ph. 3. after 30m., about no glycogen; Ph. 4. after. 1.5h., a little recover; Ph. 5. after 3h., almost recover)

D) The influence of sulfonylurea on Langerhans islet of rabbits.

We observed histology of Langerhans islet of rabbits, which were given sulfonylurea per os pro Ng. 0.25 g. Already from 15 minutes after giving medicine, stimulated status of beta cells of Langerhans islet was observed. This stimulated status is most distinct after 


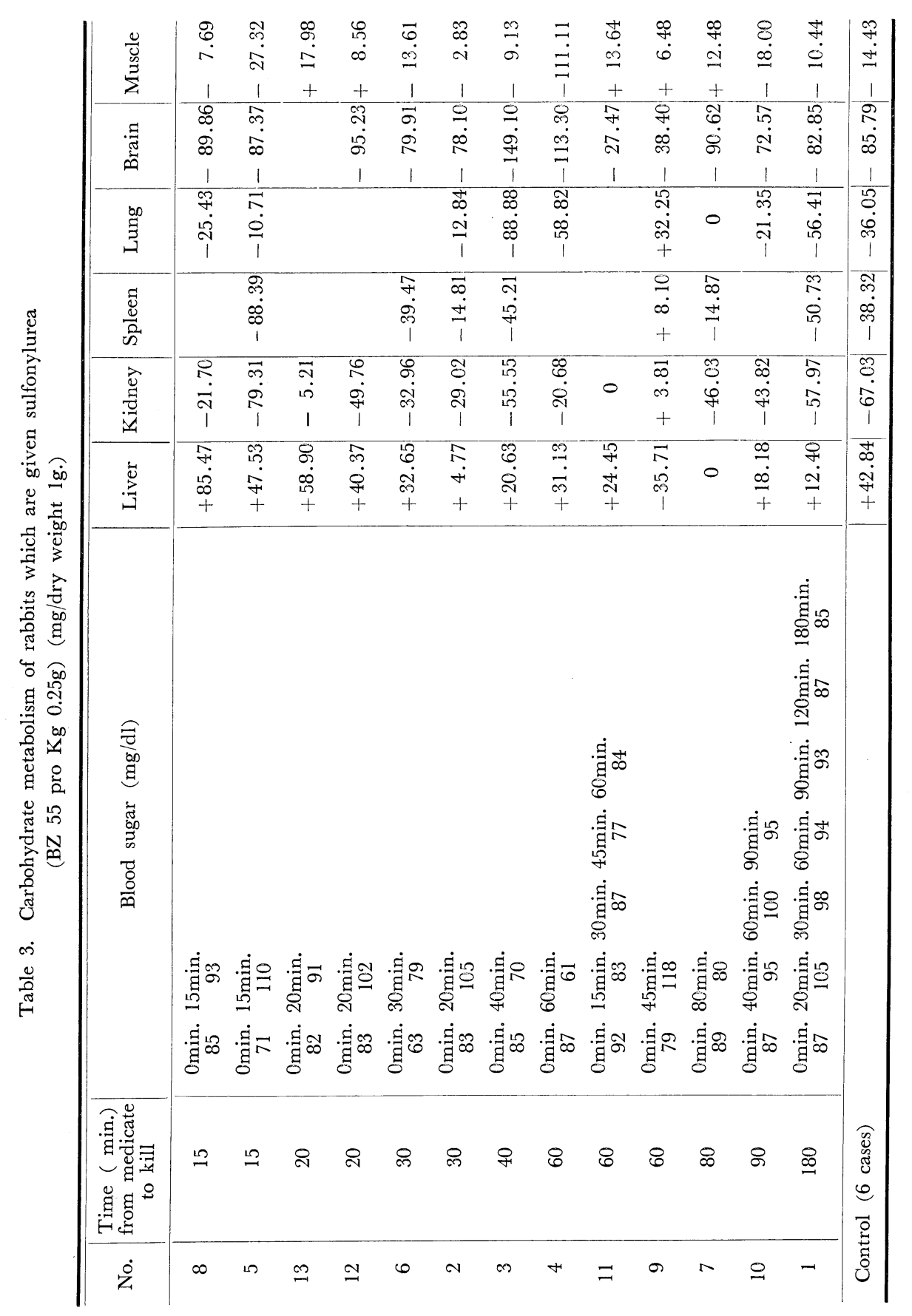


Table 4. The effect of serum of rabbit, which is given sulfonylurea on the carbohydrate metabolism of rats.

\begin{tabular}{|c|c|c|c|c|c|c|c|c|c|c|}
\hline & No. & no serum & \multicolumn{3}{|c|}{ with normal serum } & \multicolumn{3}{|c|}{$\begin{array}{l}\text { with serum at } 15 \mathrm{~min} \\
\text { after medicate }\end{array}$} & \multicolumn{2}{|c|}{$\begin{array}{c}\text { with serum at } 1.5 \text { hours } \\
\text { after medicate }\end{array}$} \\
\hline \multirow{3}{*}{$\begin{array}{l}\text { uptake of } \\
\text { diaphragm }\end{array}$} & 1 & -40.42 & \multirow{3}{*}{\multicolumn{3}{|c|}{$\begin{array}{l}-45.57 \\
-16.22 \\
-58.15 \\
\end{array}$}} & \multirow{3}{*}{\multicolumn{3}{|c|}{$\begin{array}{l}-38.76 \\
-28.55 \\
-52.07\end{array}$}} & \multirow{3}{*}{\multicolumn{2}{|c|}{$\begin{array}{l}-71.35 \\
-18.69 \\
-63.52 \\
\end{array}$}} \\
\hline & 2 & -11.47 & & & & & & & & \\
\hline & 3 & -50.08 & & & & & & & & \\
\hline \multirow{4}{*}{$\begin{array}{c}\text { output of } \\
\text { Liver }\end{array}$} & 1 & +17.00 & \multirow{3}{*}{\multicolumn{3}{|c|}{$\begin{array}{l}+14.68 \\
+\quad 6.52 \\
+57.42\end{array}$}} & \multirow{3}{*}{\multicolumn{3}{|c|}{$\begin{array}{l}+23.29 \\
+35.39 \\
+81.13\end{array}$}} & \multirow{3}{*}{\multicolumn{2}{|c|}{$\begin{array}{l}+21.80 \\
+\quad 1.78 \\
+55.55\end{array}$}} \\
\hline & 2 & +4.52 & & & & & & & & \\
\hline & 3 & +67.88 & & & & & & & & \\
\hline & No. & 0 & $15^{\prime}$ & $30^{\prime}$ & $60^{\prime}$ & $90^{\prime}$ & $2^{\circ}$ & $3^{\circ}$ & $5^{\circ}$ & $7^{\circ} \quad$ (time) \\
\hline \multirow{3}{*}{$\begin{array}{c}\text { Blood sugar } \\
\text { (mg/dl) }\end{array}$} & 1 & 103 & 131 & 126 & 128 & 121 & & & & \\
\hline & 2 & 77 & 88 & 120 & 113 & 100 & & & & \\
\hline & 3 & 81 & 98 & 90 & 125 & 86 & 83 & 92 & 75 & 73 \\
\hline
\end{tabular}

Table 5. The effect of insulin on the diaphragm.

\begin{tabular}{|c|c|c|c|c|c|c|c|c|}
\hline \multirow[b]{2}{*}{$\begin{array}{l}\text { Insulin } \\
\text { No. }\end{array}$} & \multicolumn{4}{|c|}{ uptake of glucose $(\mathrm{mg} / \mathrm{lg})$} & \multicolumn{4}{|c|}{ tissue respiration $\left(\mathrm{QO}_{2}\right)$} \\
\hline & 0 & $u / 10^{2}$ & $\mathrm{u} / 10$ & $1 \mathrm{u}$ & 0 & $\mathrm{u} / 10^{2}$ & $\mathrm{u} / 10$ & $1 \mathrm{u}$ \\
\hline 1 & -23.96 & & -24.42 & -23.00 & 6.34 & & 6.53 & 5.74 \\
\hline 4 & -27.66 & -31.69 & & -69.80 & 5.25 & 6.07 & & 8.11 \\
\hline 5 & -5.15 & -15.02 & & -31.93 & 4.95 & 5.66 & & 6.32 \\
\hline 9 & -20.95 & -21.51 & -35.32 & -22.74 & 4.99 & 6.03 & 6.10 & 4.64 \\
\hline 10 & -8.98 & & -10.35 & & 4.77 & & 5.08 & \\
\hline 11 & -14.97 & & -19.88 & & 6.19 & & 5.74 & \\
\hline 12 & -14.02 & & -27.20 & & 6.12 & & 6.33 & \\
\hline 13 & -28.82 & & -36.69 & & 7.25 & & 6.35 & \\
\hline 14 & -15.00 & & -30.03 & & 4.18 & & 6.03 & \\
\hline 15 & -19.55 & & -45.71 & & 6.20 & & 8.44 & \\
\hline 18 & -19.77 & -25.96 & -35.43 & -38.78 & 5.78 & 6.55 & 6.79 & 6.17 \\
\hline 19 & -15.89 & -29.85 & -35.02 & -40.98 & 6.91 & 6.94 & 7.08 & 6.93 \\
\hline 20 & -17.00 & -17.60 & -32.90 & -53.23 & 7.64 & 8.47 & 7.97 & 8.81 \\
\hline Medium & -17.82 & -23.60 & -30.26 & -40.06 & 5.89 & 6.62 & 6.60 & 6.67 \\
\hline
\end{tabular}

1.5 or 3 hours. These changes are observed clearly with Gomori, pyronin-methylgreen staining method, urtraviolet microscope and electron microscope.

1) Gomori staining (CHP method)

At 15 minutes after giving medicine partly, at 30 minutes after giving in almost all Langerhans islets, escape of granule of beta cells ${ }^{8)}$ and also enlargement of nucleus of beta cells are observed. These changes are observed most clearly at 3 hours after giving medicine and are observed still after 6 hours. (Ph. 6. normal; Ph. 7. after 3 hours)

Vol. 34 No. 1 
Table 6. The effect of sulfonylurea $(1 \mathrm{mg} / \mathrm{cc})$ in vitro on the tissue of rats.

\begin{tabular}{|c|c|c|c|c|c|c|c|c|c|c|c|c|}
\hline \multirow{3}{*}{$\frac{\text { Organ }}{\text { Medicine }}$} & \multicolumn{6}{|c|}{ on the carbohydrate metabolism } & \multicolumn{6}{|c|}{ on the tissue respiration } \\
\hline & \multicolumn{3}{|c|}{ Diaphragm } & \multicolumn{3}{|c|}{ Liver } & \multicolumn{3}{|c|}{ Diaphragm } & \multicolumn{3}{|c|}{ Liver } \\
\hline & 0 & BZ 55 & D 860 & 0 & BZ 55 & D 860 & 0 & EZ 55 & D 860 & 0 & BZ 55 & D 860 \\
\hline 2 & & & & 109.74 & $\begin{array}{r}108.14 \\
95.47\end{array}$ & & & & & $\begin{array}{r}9.62 \\
12.30\end{array}$ & $\begin{array}{r}10.47 \\
9.67\end{array}$ & \\
\hline 3 & & & & 120.34 & & 2276 & 455 & 560 & 4. 97 & $\begin{array}{r}12.00 \\
9.29\end{array}$ & 11.48 & 9.40 \\
\hline 6 & -18.16 & -21.13 & -25.28 & 50.38 & 45.91 & $\begin{array}{r}22.10 \\
129.12\end{array}$ & 4.55 & 0.60 & 4.97 & $\begin{array}{l}9.29 \\
0.50\end{array}$ & 1027 & 9.40 \\
\hline 7 & -10.06 & -11.46 & -17.04 & 60.12 & 93.61 & 142.13 & 6.13 & 5.34 & 6.15 & 9.50 & 10.27 & 11.33 \\
\hline 8 & -22.48 & -10.84 & -11.57 & 84.38 & 108.46 & 95.42 & 7.08 & 7.35 & 7.44 & 10.88 & 12.68 & 10.88 \\
\hline 10 & -8.98 & 0 & & 81.49 & 104.60 & & 4.77 & 5.24 & & 10.37 & 9.33 & \\
\hline 11 & -14.97 & 0 & & 105.79 & 142.54 & & 6.19 & 5.87 & & 11.06 & 10.87 & \\
\hline 12 & -14.02 & -14.33 & & 72.22 & 108.64 & & 6.12 & 6.09 & & 9.03 & 9.03 & \\
\hline 13 & -28.82 & -27.21 & & 96.29 & 84.61 & & 7.25 & 5.88 & & 11.73 & 10.56 & \\
\hline 14 & -15.00 & -39.44 & & 57.85 & 57.85 & & 4.18 & 5.07 & & 12.31 & 10.12 & \\
\hline 15 & -19.55 & -19.35 & & 72.23 & 57.75 & & 6.20 & 6.21 & & 10.40 & 9.67 & \\
\hline
\end{tabular}

Table 7. The influence of sulfonylurea $(\mathrm{mg} / \mathrm{cc})$ in vitro on the effect of insulin on the carbohydrate metabolism of diaphragm of rats.

\begin{tabular}{c|c|c|c|c|c|c|c|c}
\hline & \multicolumn{2}{|c|}{ on the carbohydrate metabolism } & \multicolumn{4}{c}{ on the tissue respiration } \\
\hline Medicine & 0 & $\begin{array}{c}\mathrm{u} / 10 \\
\text { Insulin }\end{array}$ & BZ 55 & $\begin{array}{c}\mathrm{u} / 10 \\
\text { Ins. }+ \text { BZ55 }\end{array}$ & 0 & $\begin{array}{c}\mathrm{u} / 10 \\
\text { Insulin }\end{array}$ & $\mathrm{BZ} \mathrm{55}$ & $\begin{array}{c}\mathrm{u} / 10 \\
\text { Ins. }+\mathrm{BZ55}\end{array}$ \\
\hline No. & -8.98 & -10.35 & 0 & 0 & 4.77 & 5.08 & 5.24 & 5.72 \\
10 & -14.97 & -19.88 & 0 & -17.35 & 6.19 & 5.74 & 5.87 & 6.32 \\
11 & -14.02 & -27.20 & -14.33 & -19.37 & 6.12 & 6.33 & 6.09 & 6.25 \\
12 & -28.82 & -36.69 & -27.21 & -39.54 & 7.25 & 6.35 & 5.88 & 6.91 \\
14 & -15.00 & -30.03 & -39.44 & -23.63 & 4.18 & 6.03 & 5.07 & 5.01 \\
15 & -19.55 & -45.71 & -19.35 & -20.93 & 6.20 & 8.44 & 6.21 & 7.44 \\
\hline
\end{tabular}

2) Pironin-methylgreen staining.

The more intensive staining of nucleus of beta cells with methylgreen and of protoplasma with pyronin are observed from $15 \sim 30$ minutes and clearly at 1.5 hours and less at 3 6 hours. (Ph. 8. normal; Ph. 9. after 15 m.; Ph. 10. after 1.5 hours)

3) Observation by ultra-violet microscope. $(\times 1200)$

Bausch-Lomb optical Company's ultra-violet microscope was used with $2650 \AA$ to observe nucleic acids absorption zone. Spectrum purity of this microscope is $\pm 30 \AA$.

There was remarkable reduction of nucleic acids in Langerhans' islets than that of exocrine parts and two different types of islet cells were observed. By examining the same cells in a section stained with Gomori's CHP method, their identity was established as alpha and beta cells. The nuclei of the alpha cells were dark, and those of the beta cells were pale. 
By the administration of BZ 55, after 15 30 minutes, increase inclination of ultraviolet absorption was found at beta cells' nuclei and cytoplasm. After $1 \sim 1.5$ hours absorption got maximum, and a little decreased after 3 6 hours.

The same inclination was also observed by the continued administration of BZ 55. ( $\mathrm{Ph}$. 11. normal; Ph. 12. after $15 \mathrm{~m}$. ; Ph. 13. after 1.5 h.; Ph. 14. continued administ)

4) Observation by electron microscope.

Fixation and embedding : general method.

Microtome : Sjöstrand's thermo-expansion type

Electron microscpe : Akashi's TRS-50 B type

a) On the normal rabbits

The alpha cells have a pale background cytoplasm, which contains numerous, dense, round granules. The pale nuclei are usually ovoid. One nucleolus is seen distinctly in a nucleus. (See Ph. $15 \times 15000$ )

The beta cells are darker and contain granules which are less dense and smaller than those of the alpha cells. The darker appearance of the beta cells is apparently due to an abundance of ergastoplasm in their cytoplasm. The nuclei are dark and are ovoid in shape. The other components of the cytoplasm are small, rod shaped mitochondria and a prominent Golgi complex and a fine, fibrillar substance adjacent to the nucleus. (See Ph. 16 $\times 21000)$

b) On the rabbits administrated sulfonylurea BZ 55 (after 15 minutes).

A remarkable change is not observed in alpha cells.

In beta cells the cytoplasm and the nuclei swell. The chromatine congregates now and then and becomes to some gross mass in swollen nuclei. The beta granules decrease distinctly and are found a few at periphery of the cytoplasm. A remarkable change is not observed on the mitochondria and the other components of the cytoplasm. (See Ph. 18 $\times 9000$ ) There is such a change in individual cells in various degrees on the same section. (See Ph. $17 \times 4500)$

We appreciated very much for Dr. K. Kimura's cooperation on this work.

\section{Discussion}

From our results we recognized 1)-6) about rabbits giving sulfonylurea.

1) The blood sugar first a little ascends, next descends and after a long time comes back to original value.

2) The descendence of the blood sugar of alloxan rabbits might be late.910)1112)

3) On the studium of ascendence of blood sugar output of liver glucose increases and on the studium of descendence of blood sugar glucose output of liver decreases and glucose uptake of diaphragm increases.

4) Serum of ascendence studium of blood sugar increases the glucose output of liver of other animals. Serum of descendence studium of blood sugar increases the glucose uptake of diaphragm of other animals. 
5) On the studium of ascendence of blood sugar liver glycogen decreases and there is almost no liver glycogen on the studium of hyperglycemy and on the studium of descendence of blood sugar liver glycogen increases gradually. ${ }^{13) 14) 15) 16) 17)}$

6) Already from 15 minutes after giving medicine, stimulated status of beta cells of Langerhans islet was observed. This stimulated status is most distinct after 1.5 or 3 hours. These changes are observed clearly with Gomori, pyronin-methylgreen staining method, urtraviolet ray microscope and electron microscope.

From our results we recognized 7)-8) by the addition of sulfonylurea in vitro.

7) The glucose uptake of diaphragm does not rise by the addition of sulfonylurea in vitro. ${ }^{18)(19) 20) 21)}$

8) The effect of insulin on the glucose uptake of diaphragm does not rise by the addition of sulfonylurea in vitro.

In addition to the above-mentioned results, we have the following reports :

9) There is no effect of sulfonylurea on the pancreatectomized $\operatorname{dog} .{ }^{22)-27}$ ) 3i)

10) By the big dose of sulfonylurea the necrosis of alpha cell of Langerhans islet was recognized. ${ }^{28)-34)}$

11) We can save insulin clinically and experimentally by sulfonylurea.

12) We can not think commonly, that an artifical substance as sulfonylurea is useful on many different points of therapy of diabetes mellitus as an angel on the battle against diabetes mellitus.

By above-mentioned 12 points we have come to the following belief.

Sulfonylurea work on the Langerhans islet and let the alpha cells having glucagon to put out and a rest to take ${ }^{35)}$, and bring the hyper function of beta cells and so is useful on the therapy of diabetes mellitus.

If our belief is right, we think that the examination of islet function is possible from our result of A) 4). And on the result of A) 4) we can not see the studium of ascendence of blood sugar by a little dose.

$\mathrm{Up}$ to this time there are many inverted results of the researchers throughout the world on the various studies of animals administrated sulfonylurea. We think it becomes by no attention to the up and down curve of blood sugar that changes by dose of medicine.

We have no result and can say nothing about the influence of sulfonylurea on the destruction of insulin ${ }^{936)}$.

\section{Conclusion}

we think that sulfonylurea are useful on the therapy of diabetes mellitus, by the working on Langerhans islet. And at the same time we advance the possibility of examination of islet funcaion. 


\section{References}

1) Banting, G. and Best, H. : J. Lab. Clin. Med. 1922, 464.

2) Janbon, M. et al : Montpellier Med. 21-22; 489, 1942.

3) Murlin, J. R. et al : J.B.C. 15; 365, 1913. ; 56 ; 253, 1923.

4) Bürger, M., Kramer, H. : Z. ges. exp. Med. 65; 487, 1929.

5) Bürger. M., Brandt, w. : Z. ges. exp. Med. 67; 441, 1929 ; 69 ; 57, 1930.

6) Sutherland, E.W. \& Cori, C.F. : J.B.C. 172 ; 137, 1948.

7) Gemill C.L. : Bull. Johns Hopkins Hosp. 66 ; 232, 1940.

8) Loubatieres, A. : Compt rend. Soc. biol. $138 ; 766,1944$.

9) Mirsky, I.A. et al : Metabolism 5; 138 156, 1956.

10) Beringer, A. u Lindner, A. : Wien Med. Wschr. 68; 516, 1956.

11) Chen, K.K., Anderson, R.C. and Maze, N. : proc. Soc. Exp. Biol \& Med. 63; 483, 1946.

12) Baender, A. : Deut. Med. Wschr. 81 ; 889, 1956.

13) Tyberghein, J.M. et al : Proc. Soc. exp. Biol. \& Med. 92; 322, 1956.

14) Lang, S. et al : Metabolism 5; 733, 1956.

15) Dulin, E.W. et al : Ann N.Y. Acad. Sci. 71; 177, 1957.

16) Beringer, A. u Keibl, E. : Wien. med. Wschr. 38-39; 792, 1956.

17) Miller, W. L. Jr. and Dulin W.E. : Science 123 ; 584, 1956.

18) Levine, R. : Diabetes $5 ; 375,1956$.

19) Levine, R. et al : Metabolism 5; 721, 1956.

20) Fritz, I.B. et al : Metabolism 5; 744, 1956.

21) Wick, A.N., Britton, B. and Grabownki, R. : Metabolism 5; 739, 1956.

22) Loubatieres, A. : Presse med. 63; 1701, 1728, 1955.

23) Sirek, A. : Canad. M.A.J. 74 ; 960, 1956.

24) Campbell, J. : Canad. M.A.J. 74; 962, 1956.

25) Schambye, P. : Diabetes $6 ; 146,1957$.

26) Laubatieres, A. et al : Ann. N.Y. Acad. Sci. 71; 192, 1957.

27) Houssay, B.A. et al : Ann. N.Y. Acad. Sci. 71; 12, 25, 1957.

28) Von Holt, C. et al : Arch. Exp. path. pharmac. 224; 78, 1955.

29) Ferner, H. : Deut. gesam. Pharmakol. 1955.

30) Bertram, F. et al : Deut. med. Wschr. $80 ; 1455,1955$.

31) Achelis, J.D. et al : Deut. med. Wschr. 80 ; 1452, 1955.

32) Yoshioka, H. : Naibunpi 3; 97, 1956.

33) Creutzfeldt, W. : Diabetes 6; 135, 1957.

34) Colwell, A.R. and Colwell, J.A. : Metabolism 5; 749, 1956.

35) Anderson, G.E. et al : Proc. Soc. exp. Biol. 92; 340, 1956.

36) Volk, B.W. et al : Ann. N.Y. Acad. Sci. 71; 141, 1957.

37) Yoshida, H, et al : will be published on Fol. Endocri. Jap. 